\title{
Proceedings of the Meeting of The Association of British Neurologists, United Medical and Dental Schools of Guy's and St Thomas's Hospitals, London, October 1990
}

A DIAGNOSTIC ASSAY FOR CALCIUM CHANNEL AUTOANTIBODIES IN THE LAMBERT-EATON MYASTHENIC SYNDROME (LEMS)

K Leys, B Lang, J Newsom-Davis. University of Oxford, Oxford

Passive transfer studies in mice have shown that LEMS plasma contains IgG autoantibodies that reduce the impulse-evoked release of acetylcholine by decreasing the number of voltage-gated calcium channels (VGCCs) at motor nerve terminals. The use of a radioimmunoassay for the detection of anti-VGCC antibodies in serum is described.

Cell membranes prepared from a human neuroblastoma cell line (SKN-SH) were used as the source of VGCCs; these were labelled with ${ }^{125} \mathrm{I}$-omega-Conotoxin, a component of the toxin of a fish-eating snail. Sera tested were from NCD (no cancer detected)LEMS, SCLC (small cell lung carcinoma)LEMS, SCLC without LEMS and control groups.

Taking as the upper limit of normal an antiVGCC titre of $29 \mathrm{pmol} / 1$ (mean + $(3 \times$ SD) $)$ of healthy controls $(n=10)$ and other neurological diseases $(n=8)$, we found anti-VGCC antibodies at raised titre in 16/36 $(44 \%)$ of all LEMS patients (NCD-LEMS $11 / 18(61 \%)$, SCLC-LEMS 5/18 (28\%)), $2 /$ $10(20 \%)$ of SCLC alone, and $9 / 20(45 \%)$ of immunological controls. Two patients followed for six and 11 years showed a clear inverse relationship between an electromyographic index of disease severity and anti-VGCC titres.

Although at present it detects anti-VGCC antibodies at raised titre only in a proportion of LEMS patients and in some with other immunological disorders, this assay should nevertheless prove useful in diagnosis.

PATHOPHYSIOLOGY OF FASCICULATIONS IN MOTOR NEURON DISEASE (MND). CONTRIBUTION OF MACRO EMG (MAC) RECORDINGS

RJ Guiloff, H Modarres-Sadeghi. Westminster Hospital, Charing Cross and Westminster Medical School, London

Fasciculations (FUs) were described by Denny-Brown and Pennybacker as involuntary contractions of muscle fibres belonging to one motor unit (MU) by using a concentric needle electrode and twitch recordings; several FU in MND could be evoked at will "early in slight voluntary movement". The prevailing opinion is that the generation sites of most FU are in the terminal axonal arborisation or in the presynaptic terminals of the MU. MAC allows simultaneous recordings of single fibres (SF) and most muscle fibres of the same $M U$.

A mean of 20 voluntary activated MUs (VUs) and 21 FUs were recorded in the biceps of each of 10 patients. 22 of the FUs, in nine patients, could also be activated voluntarily; their mean MAC amplitude and area were similar to that of other FUs and VUs suggesting that the sources of the potentials, and the electrode location, within the MU territory were, on average, also similar. FUs that could not be activated voluntarily had a significantly higher mean number of spikes in their triggering SF potential than the other types of units $(\mathrm{p}<0.001)$. A positive correlation between fibre density and MAC median amplitude $(\mathrm{n}=10, \mathrm{r}=0.79, \mathrm{p}=0.01)$ was found for FUs but not for VUs.

At least $10 \%$ of FUs may have generation sites near or above the point of axonal branching. Some FUs that are not activated voluntarily may have a higher number of smaller muscle fibres, or more closely packed fibres of similar of greater size, than VUs. Differences in the peripheral microanatomy of a number of FUs may underlie distal ectopic impulse generation.

MOTOR STUDIES IN HEREDITARY SPASTIC PARAPLEGIA

W Schady, JPR Dick. Manchester Royal Infirmary, Manchester

Hereditary spastic paraplegia (HSP) is a dominantly inherited disorder which causes marked lower limb spasticity but few other signs. Investigations are usually negative. We have undertaken central motor conduction (CMC) studies in 25 patients from 17 families with HSP. Fifteen patients were of juvenile onset and 9 patients were of late onset. Results were compared with those of 30 healthy subjects.

Transcranial magnetic stimulation of the motor cortex was used to evoke compound muscle action potentials from hand and leg muscles. Responses in the lower limbs were absent in 8 patients, were reduced in 1 , and were centrally delayed in $75 \%$ of patients in whom they were recordable. Responses from the hand muscles were virtually normal except for those from the five members of one family, which were considerably delayed. CMC studies did not help to discriminate between patients with and without upper limb hyperreflexia. There was no correlation between CMC parameters and clinical features such as age of onset, or duration of disease.

We conclude that HSP is a disease predominantly of the lower spinal cord. CMC studies are not useful in detecting subclinical lesions in HSP but may be of value in identifying subgroups of the disease.

THE NEURAL BASIS OF CONGENITAL MIRROR MOVEMENTS

DA Ingram, SF Farmer, JA Stephens, SW Roche, M Swash. The Royal London Hospital and University College, London

Mirror movements are recognised during willed movements of a hand muscle by the appearance of obligate activation of the homologous muscle in the opposite hand. They may occur in association with various congenital disorders and following acquired central motor lesions in adult life. No mechanism for the generation of these movements has hitherto been identified. Congenital forms are generally better defined which suggests that the two types may have a different basis. Evidence is presented for the physiological mechanism subserving congenital mirror movements in a patient aged 50 with Klippel-Feil (KF) syndrome and a patient aged 33 with familial mirroring.

In the KF patient focal percutaneous magnetic brain stimulation elicited bilateral and symmetrical short latency hand muscle responses. Cross-correlation analysis of ongoing EMG activity in homologous muscles of both hands revealed a narrow peak centred around time zero. This peak implies motor unit synchronisation due to common-stem presynaptic fibres that have branched to innervate both sides of the cord. Long latency cutaneous reflexes were distributed bilaterally, supporting their transcortical pathway. In the familial case focal brain stimulation elicited bilateral responses. In four adult patients with post-stroke mirror movements and in 10 normal control subjects only contralateral responses were elicited by focal brain stimulation and cross-correlation analysis revealed no evidence of motor unit synchronisation across the cord.

It is concluded that these congenital mirror movements result from abnormally branched corticospinal tract fibres supplying both sides of the cord. They can be differentiated electrophysiologically from the acquired form.

INTEGRITY OF CENTRAL MOTOR PATHWAYS IN STROKE PATIENTS INVESTIGATED WITH ELECTROMAGNETIC STIMULATION

A Heald, D Bates, NEF Cartlidge, J French, $S$ Miller. University of Newcastle upon Tyne, Newcastle upon Tyne

In this longitudinal study the integrity of central motor pathways has been investigated in stroke patients to determine the value of electromagnetic brain stimulation in predicting outcome. Eighty stroke patients (42-90 years) have been examined at intervals of one, three, seven, and 28 days, three and six months after stroke and 40 of these have also been reviewed at one year. Neurological and functional assessments have been compared with recordings of the surface electromyogram in muscles of the arm following electromagnetic stimulation of cerebral cortex and cervical spinal motor roots. Subtraction of onset latencies to cervical stimulation from those to cortical stimulation provides estimates of central motor conduction delay (CMCD) and the integrity of central motor pathways.

Three groups of patients can be distinguished by response to stimulation of the damaged hemisphere: those with normal CMCD, prolonged CMCD and no response to brain stimulation. The groups with prolonged CMCD or no response show poor functional recovery. Abnormal CMCD is correlated functionally with poor recovery of motor power and impaired manual dexterity. 
Individuals at high risk of poor functional outcome can be identified by measurement of CMCD within the first week after stroke.

GENE TRANSFER STUDIES ON HUMAN DYSTROPHIN

G Dickson, D Peck, D Love, KE Davies, FS Walsh. Guy's Hospital, London and Institute of Molecular Medicine, Oxford

Duchenne muscular dystrophy (DMD) is caused by a defective gene located on the $\mathrm{X}$ chromosome. Dystrophin, the protein of this gene is thought to be a membrane cytoskeletal component involved in anchoring sarcolemmal proteins to the underlying cytoskeleton, but the exact function of dystrophin and its role in the resultant necrosis of dystrophic muscle fibres have not been determined experimentally. To examine structure/ function relationships within the predicted sub domains of dystrophin we have prepared human cDNA gene constructs and have transfected muscle and non-muscle cells in vitro. Using a cDNA encoding the $\mathrm{N}$ terminal putative actin-binding domain of dystrophin under control of the murine leukaemia virus promoter, expression of an appropriate mRNA and of truncated human dystrophin was obtained in mouse fibroblast and myoblast cells. In contrast to the native molecule, the truncated recombinant dystrophin exhibited a perinuclear localisation associated with filamentous cytoplasmic network. This observation supports the predic ted cytoskeletal-binding role of this part of the dystrophin molecule and indicates that elements involved in membrane-association are located towards the carboxy-terminus.

ANALYSIS OF DNA DELETIONS IN DUCHENNE AND BECKER MUSCULAR DYSTROPHY

SV Hodgson, S Abbs, SC Yau, S Clark, CG Mathew, V Dubovitz, JZ Heckmatt, E Rodillo, M Bobrow. Guy's Hospital and Hammersmith Hospital, London

In $50-60 \%$ of cases of Duchenne (DMD) and Becker (BMD) muscular dystrophy, a deletion can be detected within the dystrophin gene, by failure of hydridisation of patient DNA to cloned CDNA sequences. We have analysed over 400 cases of $D / B M D$, and correlated deletion findings with clinical features. We divided affected males into three groups-(1:DMD), in which ambulation was lost before 13 years; (2: intermediate), where walking ceased between 13 and 16 years, and (3: BMD), in which ambulation continued beyond 16 years.

A deletion was found in 222 cases (55\%), and the extent was delineated using cDNA probes. The number of exons deleted was independent of phenotype. Certain deletions, however, were correlated with disease severity. Deletion of exons 3-7 occurred in six patients with the intermediate phenotype, and in two patients with BMD. Certain other small deletions were specific for BMD, although large deletions were also found in some boys with BMD. We have detected one unusual deletion in a boy with atypical clinical presentation.

Approximately $98 \%$ of pathogenic deletions in the dystrophin gene can now be detected, using a rapid technique that involves the simultaneous analysis of several exons using the polymerase chain reaction.
INCIDENCE AND CLASSIFICATION OF PERIPHERAL NERVE SYNDROMES IN HIV INFECTION. A PROSPECTIVE STUDY GN Fuller, JM Jacobs, RJ Guiloff. Westminster Hospital, Charing Cross and Westminster Medical School, and Institute of Neurology, London

In a 15 month study 56 patients with nontoxic peripheral nerve syndromes were seen out of a population of 1353 HIV positive patients followed in Riverside, of whom 261 had AIDS and 748 had AIDS-related complex (ARC). Distal symmetrical peripheral neuropathies (DSPN) were most commonly seen, all in patients with AIDS, 25 having pain (PPN) and 13 without. Twelve patients were seen with isolated mononeuropathies, (nine with AIDS and three with ARC), three with mononeuritis multiplex (two due to lymphoma and one ARC with vasculitis). Two AIDS patients had lumbrosacral polyradiculopathy. There were no patients with acute or chronic inflammatory demyelinating peripheral neuropathy.

Estimates of the incidence can be made on the basis of the numbers of patients at risk of each complication. In AIDS the incidence of PPN was $10 \%$, other DSPN 6\%, lumbosacral polyradiculopathy $0.8 \%$, lymphomatous mononeuritis multiplex $0.8 \%$ and other mononeuropathies $3.6 \%$. In ARC and AIDS vasculitis occurred in $0.1 \%$. In ARC mononeuropathies were seen in $0.4 \%$.

These data support the classification of peripheral nerve syndrome in HIV infection according to the stage of the disease. They cast doubts on the reported association of inflammatory demyelinating neuropathies and HIV infection.

SUBACUTE COMBINED DEGENERATION WITH HIGH SERUM VITAMIN B12: NEW CAUSE OF AN OLD SYNDROME

EH Reynolds, T Bottiglieri, J Stern, J Payan, J Linnell, J Faludy. King's College Hospital and Westminster Hospital, London

A 29 year old male presented with severe megaloblastic anaemia which required transfusion. As his serum vitamin B12 was very high $(>1800 \mathrm{pg} / \mathrm{ml})$ and red cell folate low $(82 \mathrm{ng} / \mathrm{ml})$ he was treated with folic acid, but he remained macrocytic $(\mathrm{MCV}>100 \mathrm{fl}$ ). Three years later he presented with subacute combined degeneration, a sensory axonal neuropathy and seizures. Serum vitamin B12 remained very high, serum and red cell folate were very high (on treatment) but red cell vitamin B12 level was very low and he was found to have an abnormal binder for vitamin B12 in his plasma. In vitro experiments indicated that the abnormal binder limited the entry of vitamin B12 into his cells, thus accumulating vitamin B12 in the serum. Folic acid was withdrawn and he was later treated with intensive vitamin B12, at which point his blood count returned to normal. Neurologically and electrophysiologically he has made an almost complete recovery.

A SURVEY OF UNDERGRADUATE TEACHING OF CLINICAL NEUROLOGY IN THE UNITED KINGDOM, 1990

IMS Wilkinson. Addenbrooke's Hospital, Cambridge

Clinical relevance has a low profile in the neurological teaching of medical students during the preclinical phase of their course.
A formal attachment of all clinical students to the department of neurology occurs in 20 of the 28 UK medical schools. Such attachments are, on average, either three to four weeks full time or six weeks part time. Sharing with neurosurgery is very successful.

Learning the technique of neurological examination is satisfactory in $75 \%$ of schools. During the clinical attachment, exposure to the common neurological diseases and emergencies, and to chronic neurological disability, is sufficient in only $50 \%$ of schools.

Even though a comprehensive lecture series given by neurologists to clinical students occurs in 19 of the 28 schools, clinical based teaching remains highly valued as the best way to learn neurology.

Twenty of the 28 schools have a ratio of clinical students to teaching consultant neurologists of $28: 1$ or less. In these schools, staff feel their numbers are adequate and a clinical attachment in neurology is offered to all students. In the remaining eight schools, where the ratio is greater than 28:1 staff are unable to meet the teaching need, and only a fraction of students have a clinical neurological attachment.

\section{INTELLECTUAL IMPAIRMENT IN}

NEUROFIBROMATOSIS 1 (NF1)

RE Ferner, RAC Hughes. United Medical and Dental Schools, Guy's Hospital, London

Intellectual problems are common in NF1 but their aetiology is unknown. Neurological and psychometric assessments were performed on 100 NF1 patients and 100 age, sex and socio-economically matched controls. Thirty patients had MRI head scans. The NF1 group had significantly lower IQ scores than the controls with full scale IQ 88.5 (controls $101 \cdot 8$ ), verbal IQ 90.5 (controls $102 \cdot 4$ ), and performance IQ 88.1 (controls 101.5). Eight per cent of patients and no controls had an IQ score less than 70 . Thirty four per cent of patients and $4 \%$ of controls had received remedial education. The patients performed significantly worse on computerised attention tasks, and demonstrated poor memory recall and reading ability. Anxiety and depression scores did not differ significantly between the two groups. The NF1 group showed a drift down in socio-economic status in relation to their parents. T2 weighted MRI sequences showed increased intensity lesions, possibly haematomas in the basal ganglia, cerebellum and corona radiata in four patients. Abnormalities on MRI scans did not correlate with IQ. It was concluded that NF1 patients have significant intellectual impairment, compared with a matched control group. No relation was shown between intellectual deficits and structural abnormalities in the brain, disease severity or psychiatric problems.

THE NATURE OF THE NAMING DEFICIT IN ALZHEIMER'S DISEASE

JR Hodges, DP Salmon, N Butters. Cambridge University, Cambridge and University of California, San Diego, USA

Although progressive anomia is a well recognised feature of Alzheimer's type dementia (DAT) the nature of the underlying deficit remains uncertain. We compared the naming performance of 52 patients with DAT and 54 normal control subjects using the Boston Naming Test. All errors were categorised using a comprehensive system of classification 
derived from experimental cognitive psychology models of naming.

The DAT patients' spontaneous and cued naming scores were significantly impaired $(p<001)$. Normal controls made predominantly semantic-category and circumlocutory errors. By contrast, the patients made a significantly greater proportion of semantic-superordinate and semanticassociative errors $(p<\cdot 05)$. A subgroup of 22 patients were followed longitudinally over three years. Their deterioration in overall naming performance was accompanied by a consistent change in the profile of naming errors: the proportion of semantic-associative errors increased significantly as did the proportion of visual errors $(p<\cdot 05)$.

These results suggest that in DAT the primary deficit underlying the anomia is a breakdown in semantic knowledge. As the disease progresses, however, perceptual problems also begin to contribute to the patients' naming difficulties. Post-lexical (phonemic) processes remain relatively intact throughout.

CLINICAL PATHOLOGICAL CORRELATIONS OF LOBAR ATROPHY

PN Cooper, DM Mann, JS Snowden, PJ Goulding, D Neary. Manchester Royal Infirmary, Manchester

Lobar atrophy is a genetically determined cerebral degeneration in which the topographical distribution of pathological change determines the nature of the neuropsychological syndrome which comprise dementia of frontal lobe type (DFT), DFT and motor neuron disease (MND) and progressive aphasia. Brains from such patients have permitted clinico-pathological correlations. In DFT progressive behavioral disturbance is followed by speech impairment and mutism. Cerebral atrophy principally affects the frontal and temporal lobes, grey and white matter being affected equally and also the corpus striatum and thalamus.

In DFT and MND, rapidly progressive dementia is followed by bulbar palsy and amyotrophy and death within two years. The distribution of atrophy is similar to DFT but is milder in degree. In addition, there is severe degeneration of the substantia nigra, the hypoglossal nuclei and the anterior horn cells of the spinal cord.

In progressive aphasia, speech impairment advances to mutism in the relative absence of other neuropsychological disorders. There is atrophy of the fronto-parietal and temporal lobes of the left cerebral hemisphere with degeneration of the corpus striatum and thalamus. There is moderate right temporal atrophy only.

These findings confirm lobar atrophy as a form of cerebral atrophy distinct from $\mathrm{Alz}$ heimer's disease.

JUVENILE MYOCLONIC EPILEPSY I. CLINICAL ASPECTS

CP Panayiotopoulos, T Obeid. St Thomas' Hospital, London and King Khalid University Hospital, Riyadh

This was a prospective clinico-electroencephalographic study of 66 patients with juvenile myoclonic epilepsy (JME). There were 34 male and 32 female patients (ratio $=1 \cdot 06$ ). Sixty three of the patients were not diagnosed on referral and 22 of them were not initially recognised in the epilepsy clinic.
Clinical absences were reported in 22 $(33.3 \%)$, myoclonic jerks in $64(97.0 \%)$ and generalised tonic-clonic seizures (GTCS) in $52(78.8 \%)$ of the patients. The majority of them (38 patients, $57.6 \%$ ) had myoclonic jerks and GTCS

The mean (SD) age of onset was $10.5(3.4)$ (range 5-16) years for absences, 15 (3.5) (826) for myoclonic jerks and 15.2 (3.6) (8-26) for GTCS. Absences predated myoclonic jerks and GTCS for $3.9(2.3)$ (range 1-9, median 5) and $4 \cdot 4(2 \cdot 7)$ (range 1-8, median 8 ) years respectively in 14 patients who manifested the triad of these seizures $(21 \cdot 2 \%)$. Absences were never preceded by myoclonic jerks or GTCS.

Myoclonic jerks had a characteristic circadian distribution on awakening in 56 $(87.5 \%)$ of the patients. GTCS were mainly on awakening ( 11 out of 16 patients, $68 \cdot 75 \%$ ). In two patients GTCS were nocturnal only. Neurological examination was normal for all patients except tremor of the hands similar to the idiopathic familial tremor which was found in $23(35 \%)$ patients. CT brain scan performed in 23 patients was normal.

JUVENILE MYOCLONIC EPILEPSY II.

PRECIPITATING FACTORS, GENETICS, EEG FINDINGS AND TREATMENT

CP Panayiotopoulos, T Obeid. St Thomas' Hospital, London and King Khalid University Hospital, Riyadh

This was part II of a prospective clinico-EEG study of 66 patients with juvenile myoclonic epilepsy (JME). Precipitating factors were found in all but $4(6.2 \%)$ patients. These were sleep deprivation $(78.5 \%)$, fatigue $(64.4 \%)$, photic stimulation $(33.8 \%)$, menstruation $(21.9 \%$ of women), mental concentration $(20 \%)$, stress-expectation $(10 \cdot 8 \%)$ and visual display systems $(6 \cdot 2 \%)$.

The high incidence of JME amongst siblings (13 families) with only one family with maternal involvement favours an autosomal recessive mode of inheritance.

Normal EEGs were frequently recorded in treated patients. However, at least one abnormal EEG was recorded in $56(84.9 \%)$ patients. The abnormalities consisted mainly of generalised discharges of spike/double spike and/or polyspike and slow wave. There were frequent multiple spike like compressed Ws and discharge fragmentations. Their duration varied from $0 \cdot 5-20 \mathrm{~s}$. Focal abnormalities were seen in $20(30.3 \%)$ patients. Photoconvulsive discharges were recorded in $18(27.3 \%)$ patients.

Video-EEG recordings and clinical observations indicate that typical absences of JME have a characteristic clinico-EEG pattern which differentiates them from those of childhood, juvenile and myoclonic absence syndromes.

Treatment was mainly effective $(80 \%$ of the patients) with sodium valproate. Clonazepam may be added for a better control of the myoclonic jerks. Clonazepam monotherapy does not prevent generalised tonic-clonic seizures.

THE CONSEQUENCES OF REPERFUSION AFTER STROKE

JV Bowler, JPH Wade, BE Jones, RF Jewkes, TJ Steiner. Charing Cross Hospital, London

Thrombolysis is a potential treatment for stroke and clarification of the natural history of reperfusion following stroke is needed.
This was assessed using the Charing Cross Stroke study in which 136 serial SPECT scans were performed prospectively on 60 consecutive strokes. The first scan was done at about 24 hours and repeated at one week and three months. In addition CT was performed to confirm the lesion's site and degree of haemorrhagic infarction (HI). Full noninvasive investigations were performed to determine the aetiology.

Forty five cases were classifiable as embolism or thrombosis. Reperfusion was seen in $10 / 19$ emboli and 5/26 thromboses ( $p=0.028$; Chi square with Yates correction). HI was associated with embolism, being seen in 7/19 emboli and 2/26 thromboses $(p=0.031)$, and with reperfusion being seen in $6 / 15$ reperfused lesions and only $3 / 30$ non-reperfused lesions $(p=0.037)$. However, reperfusion was only seen once at 24 hours. Only then did it improve outcome. Reperfusion did not otherwise affect outcome as determined by stroke score, infarct volume or Barthel score. Though strongly associated (8/9) with HI $(p=0.0002)$ significant oedema was not associated with reperfusion suggesting a different pathogenesis.

LACUNAR TIAS: THEY MUST EXIST, BUT CAN THEY BE RECOGNISED AND DO THEY HAVE CLINICAL RELEVANCE?

GJ Hankey, CP Warlow. Western General Hospital, Edinburgh

Lacunar ischaemic stroke syndromes can be distinguished clinically, pathophysiologically and prognostically from cortical ischaemic stroke syndromes. The authors wished to test the hypothesis that transient ischaemic attacks (TIAs) share similar heterogeneity and that lacunar TIAs can be distinguished from cortical TIAs. If a right handed patient experiences transient right-sided symptoms and also attempts to speak during the TIA then it may be possible to infer whether the TIA involved the middle cerebral artery cortical branches ("cortical TIA") or penetrating lenticulostriate branches ("lacunar TIA").

One hundred and thirty patients who met these criteria, of whom 71 had carotid angiography were prospectively studied. Internal carotid artery stenosis of $\geq 50 \%$ was present on the symptomatic side in $36(67 \%)$ of the 54 patients who were considered to have cortical TIAs but only one $(6 \%)$ of the 17 patients with lacunar TIAs $(p<0.0001$ ) These findings support the hypothesis that patients with cortical TIAs have large (carotid) artery disease (that is, atheroma) and that the patients with "lacunar TIAs" have absent or insignificant ipsilateral extracranial carotid artery disease, and probable intracranial small vessel disease. The capacity to distinguish between lacunar and cortical TIAs may have implications for patient management.

THE MECHANISM OF CLINICAL IMPROVEMENT FOLLOWING FETAL TRANSPLANTATION FOR PARKINSON'S DISEASE: EVIDENCE FROM POSITRON EMISSION TOMOGRAPHY STUDIES GV Sawle, DJ Brooks, O Lindvall, P Bloomfield, CD Marsden, RSJ Frackowiak. MRC Cyclotron Unit, Hammersmith Hospital, The National Hospital for Nervous Diseases, London and University Hospital, Lund, Sweden

Positron emission tomography (PET) and 
$\left[{ }^{18} \mathrm{~F}\right]-6$-L-fluorodopa were used to study two patients before and after fetal mesencephalic implantation for Parkinson's disease.

In the first patient, serial PET scans performed 12 months before and five, eight and 13 months after operation, demonstrated a progressive increase in tracer accumulation in the anterior and middle portions of the transplanted putamen. (Left/Right Caudate ratio $0.84,0.89,0.88$ and 0.82 , Left/Right Putamen ratio $0.97,1.26,1.39$ and 2.00 , at $-12,5,8$ and 13 months pre/post engraftment). The second patient also demonstrated a progressive but more localised increase in signal intensity in the operated putamen between 12 months pre-operation, seven and 12 months post-operation.

This progressive change in signal activity contrasts with the patients' performance on clinical assessment. Both improved clinically two to three months after operation. In the first case a major reduction in symptomatology was recorded, whilst in the second patient changes were modest but nevertheless significant. Although in both cases the improvement observed at two to three months has been sustained, neither patient has improved further.

The dissociation between clinical and PET findings may suggest that the maximal clinical improvement from this operation depends more upon the anatomical extent of successful dopaminergic reinnervation than the degree of dopaminergic function within surviving graft tissue.

DOES ABNORMAL STIMULUS PROCESSING CONTRIBUTE TO DELAYED MOTOR RESPONSE IN PARKINSON'S DISEASE?

CD Ward, SJ Cowell, D Marcer, WA Matthews. University of Southampton, Southampton

There is clinical and experimental evidence suggesting that attentional deficits might contribute to motor impairment in Parkinson's disease (PD). Goodrich et al (1989) found that in control subjects simple reaction time (SRT) was prolonged when combined with a secondary attention-demanding task which had no effect on choice reaction time (CRT). In PD patients there was a specific impairment of SRT (but not of CRT); and the SRT deficit was not increased by the secondary task. They proposed that the normal speed advantage in SRT depends on an attentiondemanding process which is impaired in PD. Alternatively the SRT stimulus might be over-processed in $\mathrm{PD}$, with aquisition of redundant stimulus characteristics. The authors studied the extent of stimulus processing using words as stimuli, and using indices of implicit verbal learning as measures of incidental processing (given that the meaning and structure of the words were irrelevant to the task). A balanced cross-over design was used in 20 patients and 20 matched controls. We confirmed the specific SRT deficit in PD but found the extent of incidental stimulus processing to be neither decreased nor increased compared with controls. Unexpectedly, both groups showed more irrelevan processing of SRT than of CRT stimuli, indicating that attentional processes in the two tasks are fundamentally different. However, there is no evidence that the SRT deficit in PD is due to abnormal stimulus processing.
DIFFERING PATTERNS OF DISRUPTION OF THE STRIATAL PRE- AND POST-SYNAPTIC DOPAMINERGIC SYSTEMS IN PARKINSON'S DISEASE, MULTIPLE SYSTEM ATROPHY, AND PROGRESSIVE SUPRANUCLEAR PALSY

DJ Brooks, V Ibanez, GV Sawle, D Playford, N Quinn, CJ Mathias, R Bannister, AJ Lees, CD Marsden, RSJ Frackowiak. MRC Cyclotron Unit, Hammersmith Hospital and the National Hospital for Nervous Diseases, London

Striatal uptake of ${ }^{18} \mathrm{~F}$-Dopa and ${ }^{11} \mathrm{C}$ raclopride reflects the integrity of nigrostriatal dopaminergic fibres, and striatal $\mathrm{D}_{2}$ dopamine binding sites, respectively. PET was performed on 16 patients with levodopa responsive Parkinson's disease (PD), and 28 patients with non levodopa responsive Parkinsonism [18 Multiple System Atrophy (MSA), and 10 Progressive Supranuclear Palsy (PSP)]. The three groups of PD, MSA, and PSP patients all had mean posterior putamen ${ }^{18} \mathrm{~F}$-Dopa influx constants $(\mathrm{Ki})$ that were reduced to $40 \%$ of normal. Mean caudate and anterior putamen $\mathrm{Ki}$ values were equally severely reduced in PSP, but relatively preserved in PD. MSA patients showed both mildly and severely affected caudate ${ }^{18} \mathrm{~F}$-Dopa uptake. Reduction in putamen ${ }^{18} \mathrm{~F}$-Dopa $\mathrm{Ki}$ correlated with locomotor disability in PD and MSA, but not in PSP.

Untreated PD patients had normal levels of striatal $\mathrm{D}_{2}$ receptors, but treated $P D$ patients showed a significant $30 \%$ reduction in mean caudate and putamen ${ }^{11} \mathrm{C}$-raclopride uptake. MSA and PSP patients also showed depressed striatal ${ }^{11} \mathrm{C}$-raclopride uptake, but less than the treated PD patients. It is concluded that nigro-posterior putamen dopaminergic projections are principally disrupted in PD, while the nigra is more diffusely affected in MSA and PSP. Levodopa resistance in MSA and PSP does not appear to be a consequence of loss of striatal $\mathrm{D}_{2}$ dopamine receptors.

AMBLYOPIA IN CHILDREN IMPROVED BY YELLOW FILTERS

JF Stein, MS Fowler. University of Oxford, Oxford and Royal Berkshire Hospital, Reading

Recently there has been much media interest in the possibility that giving dyslexic children tinted spectacles may help them to learn to read. The authors see approximately 500 children with reading difficulties each year; and we have been trying to find out whether coloured filters can help them. A small number of children have been identified (c. $2 \cdot 5 \%$ of new cases-average age nine years) who had reduced Snellen acuity, stereoacuity and accommodation, together with impaired contrast sensitivity; but whose poor vision was not the result of refractive errors, colour blindness, other retinal abnormalities or strabismus. Their symptoms, however, were immediately alleviated by looking through yellow filters, and after six months wearing yellow lenses in many the improvement persisted after removal of the filters. It is believed that their initial impairments may have been the result of failure to compensate adequately for chromatic aberration, in particular the blur caused by short wavelengths. Yellow filters may therefore help these children on account of their "negative blue" effect. It should be noted however that many of these children did not have serious reading problems, and successfully treating their amblyopia did not improve the reading of any of them.

DETECTION OF ABNORMALITIES IN

"UNDIAGNOSED" SPINAL CORD DISEASE USING MEMORY-SCANNING EVOKED POTENTIALS (ERP) LD Blumhardt, M Holly, L Pelosi, G Barrett, M Hayward, E Ghadiali, T Slade. University of Liverpool, Liverpool

Long-latency potentials evoked by a memory scanning task were compared with psychological tests of memory in the detection of early "silent" cognitive impairment.

Twenty five cases were randomly selected from a prospective study of isolated "undiagnosed myelopathy" suspected to be due to MS. Psychological tests identified 8/25(32\%) patients with abnormal memory. MRI lesions were present in $100 \%$ of these cases versus $71 \%$ of the rest.

Responses were evoked by "probe" digits either present (positive) or absent (negative) from a memory set of 1,3 or 5 digits presented aurally or visually.

In healthy controls, the major components of the ERP's to memory sets of one digit, were a negative wave (N1) and a following positive wave (P3a) (average latencies of $170 \mathrm{~ms}$ and $430 \mathrm{~ms}$ for the auditory presentation and $190 \mathrm{~ms}$ and $360 \mathrm{~ms}$ respectively, for the visual). Patients with abnormal memory test scores showed reduced ERP amplitudes (particularly for the auditory modality) and marked waveform alterations, with the appearance and/or enhancement of one or more broad negative waves ("N2") following N1. The inter-peak latency difference between $\mathrm{N} 1$ and "N2" was prolonged compared with healthy subjects.

Memory-scanning ERP can provide objective evidence of defective recent memory.

RESTORATION OF CONDUCTION TO DEMYELINATED CENTRAL NERVE FIBRES BY REDUCED TEMPERATURE BUT NOT BY 4AMINOPYRIDINE (4-AP)

PA Felts, KJ Smith. Eastern Virginia Medical School, Norfolk, Virginia, USA and UMDS, Guy's Campus, London

The symptomatology of multiple sclerosis (MS) can sometimes be improved by a reduction in body temperature, or the administration of 4-AP, a $\mathrm{K}^{+}$-channel blocking agent. The improvements have been attributed to a restoration of conduction in formerly blocked fibres. To examine this mechanism, focal demyelinating lesions were induced in four rats using intraspinal injections of ethidium bromide or anti-galactocerebroside serum. Half the animals were examined at multiple time points using chronically implanted electrodes, the others were examined acutely. Reduction of body temperature from $38^{\circ} \mathrm{C}$ to $33^{\circ} \mathrm{C}$ increased the number of long latency fibres conducting through the lesion during the period of demyelination, at two, and particularly, at four and six weeks post-injection. However, administration of 4-AP (1.7$2.7 \mathrm{mg} / \mathrm{kg}$, i.v.) had little or no effect on the number of conducting fibres at these, or other, times.

The observation that temperature reduction improved conduction in experimentally demyelinated fibres but that 4-AP administration did not, suggests that the beneficial effects of 4-AP in MS patients may be due to effects other than an increase in the number of 
conducting fibres, such as effects on synaptic transmission.

RESPIRATORY INVOLVEMENT IN MULTIPLE SCLEROSIS

RS Howard, NP Hirsch, CM Wiles. The National Hospital for Neurology and Neurosurgery, London

Respiratory insufficiency occurs during the advanced stages of multiple sclerosis but may also complicate acute relapses earlier in the course of the disease. The authors present 18 patients with multiple sclerosis who developed respiratory complications at a mean of 5.6 (range 1-12) years after the onset of neurological symptoms.

Thirteen patients developed severe respiratory insufficiency manifest as a combination of reduced vital capacity and hypoxia (12 patients), and respiratory arrest (1). Two patients presented with apneustic breathing, one with paroxysmal hyperventilation, one with obstructive sleep apnoea and one with bulbar weakness leading to aspiration pneumonia. Respiratory muscle weakness was a major factor in nine patients (predominantly diaphragm involvement in six), bulbar weakness in six patients, impaired voluntary control (3) and impaired automatic control (3).

Eleven patients received respiratory support of whom seven have subsequently died. The methods of support used were intermittent positive pressure ventilation ( 8 patients) iron lung (3), cuirass (2), and rocking bed (1). Six patients were maintained on respiratory support until they died after intervals ranging from 24 hours to six years (mean 17.7 months). Five patients received temporary ventilation for between six and $\mathbf{4 2}$ days following which four of these remain alive at up to four years and one died after 16 years.

NEUROLOGICAL FEATURES AND MANAGEMENT OF BLADDER DYSFUNCTION IN MULTIPLE SCLEROSIS

CD Betts, MT D'Mellow, C Fowler. The National Hospital for Neurology and Neurosurgery, London

The urinary symptoms in 150 patients with multiple sclerosis were studied. Frequency, urgency and incontinence were the commonest complaints, occuring in $\mathbf{9 8 \%}$. Hesitancy and difficulty initiating voiding were concurrent symptoms in $30 \%$. Complete retention requiring catheterisation was unusual.

All patients were scored on the Kurtzke expanded disability status scale. No correlation was found between the severity of bladder symptoms and any functional group except pyramidal function score.

Urodynamic studies were performed in $32 \%$ of the patients and detrusor hyperreflexia was demonstrated in all patients. This correlated well with symptoms of frequency and urgency. In $40 \%$ residual volumes were greater than $100 \mathrm{mls}$, but no feature of the history was found to be helpful in predicting this. In those patients in whom urodynamics were not carried out a similar incidence of a high residual volume was found. All patients capable of performing the technique were taught clean intermittent self-catheterisation. However, the majority of these patients also required anti-cholinergics to lessen frequency, as assessed by patient kept frequency volume charts.

It is concluded that optimal management of urinary symptoms in patients with multiple sclerosis can be achieved by first measuring the residual volume, instituting clean intermittent self-catheterisation where possible and treatment with anti-cholinergics if frequency and urgency persist.

FAILURE TO ATTEND NEUROLOGY CLINICS W Dickey, JI Morrow. Royal Victoria Hospital, Belfast

We studied 231 patients referred to the outpatient clinics of two consultant neurologists in Northern Ireland to determine factors associated with non-attendance. Of 188 patients referred to the regional centre 43 $(23 \%)$ did not attend, compared with $3(7 \%)$ of 43 patients referred to peripheral clinics, despite longer waiting lists in the latter. Compared with attenders, patients who failed to attend the regional centre clinics were significantly younger (mean age $33+13.9$ years v. $39 \pm 17 \cdot 8$ ). Other factors significantly associated with non-attendance were male sex ( $p \leq 0.05$ ), symptom duration less than 12 months ( $p \leq 0.05$ ), referral from Accident and Emergency Departments $(p \leq 0.01)$ and city home address $(p \leq 0.01)$. Symptoms prompting referral and length of waiting list were not significantly related to non-attendance.

The better attendance rates of patients with rural addresses, both at peripheral and regional centre clinics, and the poor attendance rate of patients referred from Accident and Emergency Departments are relevant to outpatient clinic policies.

PERIPHERAL NEUROPATHY IN SCLERODERMA W Schady, A Hassall, L Holt, M Jayson. University of Manchester, Manchester

Peripheral neuropathy in scleroderma is though to be rare. We have undertaken a quantitative assessment of peripheral nerve function in 29 patients with either the CREST syndrome or progressive systemic sclerosis. Tactile thresholds were raised in the fingers in $28 \%$ and in the foot in $50 \%$ of patients. Two-point discrimination was abnormal in 10 patients, thermal thresholds in five and vibration thresholds in one. Nerve conduction studies showed absent or reduced lower limb sensory action potentials in six patients, four of whom had clinical signs of a mild peripheral neuropathy. Their mean duration of disease (20.5 years) was longer than that of other patients ( 12.9 years). There was electrophysiological evidence of a subclinical carpal tunnel syndrome in three patients. The sympathetic skin response was recorded in the 16 patients who had not had a sympathectomy. It was abnormal (below $100 \mu \mathrm{V}$ in the hand and absent in the foot) in four patients.

These results indicate that peripheral nerve dysfunction in scleroderma, though mild, is not as uncommon as was previously thought. The abnormal cutaneous sensory thresholds may be partly due to altered viscoelastic properties of the skin, but the predominant lower limb involvement in tests of tactile sensitivity, nerve conduction and peripheral sympathetic function argues in favour of an axonal neuropathy. Distal nerve trunk ischaemia may be responsible.
ANTI-GANGLIOSIDE ANTIBODIES IN GUILLAINBARRÉ SYNDROME

M Cronin, P Doherty, S Koblar, J Winer, FS Walsh. UMDS, Guy's Hospital, London

As a first step in the analysis of autoimmune mechanisms in Guillain-Barré Syndrome (GBS) the titre of antibodies reactive with a variety of gangliosides were measured. A group of 95 GBS patients with age and sex matched controls were tested for antibodies against the purified gangliosides GM1, GD1a and GDlb by an enzyme linked immunoabsorbant assay (ELISA). Samples with binding greater than the mean plus three standard deviations of the control population were considered positive. By this criterion, none of the control population were positive, whereas 14 GBS $(15 \%$ of the GBS population) showed positive binding to GM1 and/or GD1b. Among the patients that were positive, eight showed reactivity to both GM1 and GD1b, two to GD1b alone and four to GM1 alone. The antibody class in these sera was predominantly IgG rather than IgM. These patients were also tested for reactivity to both GT1b and GM2 and in general were found to be negative, suggesting the reactivity to be against the $\mathrm{Gal}(\beta 1-3)$ GalNAc epitope common to GM1 and GD1b. Patients positive for ganglioside antibodies tended to show a poor prognosis in that six out of 13 deaths were in the subpopulation that tested positive.

COMMON HOST GLYCOLIPID IN THE ENVELOPES OF BRAIN DERIVED BUDDING VIRUSES AND THEIR ABERRANT FORMS. THE IMPLICATIONS IN VIRAL INDUCED CNS DEMYELINATION: AN IMMUNO-ELECTRON MICROSCOPICAL STUDY

S Pathak, J Illavia, A Khalili-Shirazi, HE Webb. St Thomas' Campus, UMDS, London

Semliki Forest virus (SFV) (the demyelinating strain), influenza and measles are RNA viruses replicating by budding from the host cell membrane taking host cell membrane glycolipid into their envelopes.

These viruses were grown in identical mouse brain cell cultures and using immunoelectron microscopy (IEM), were labelled with a gold conjugated anti-glycolipid monoclonal antibody directed chiefly against galactocerebroside, derived from SFV infected mice. Polyclonal anti-galactocerebroside antibody also labelled the SFV.

Interestingly the SFV infection mature virus and membrane bound spherules, the latter an aberrant form of virus which also bud from the cell membrane but are different in appearance from mature virus, were both labelled.

This IEM study shows that these mature enveloped viruses and the spherules budding from the same type of CNS cells contain a common glycolipid in their envelopes which is antigenically available and with which immunological reactions can take place. The presence of common glycolipid derived from the host cell membrane in the envelopes of mature budding viruses and their aberran forms may play a role in virus induced CNS autoimmunity and demyelination, particularly in MS with which a number of enveloped viruses have been implicated. 
IMMUNITY TO GLYCOLIPID IN MULTIPLE SCLEROSIS

M Parsons, SJ Illavia, HE Webb. St Thomas' Campus, UMDS, London

Isoelectric focusing and viral (Western) immunoblotting using measles, rubella and influenza viruses grown in vero cells have been applied to cerebrospinal fluid (CSF) from multiple sclerosis (MS) patients Individual MS CSF blots showed no response to rubella, but measles and influenza viruses picked out common peaks in the oligoclonal IgG pattern. These common peaks must relate to IgG which recognises antigens common to both viruses. Enveloped viruses bud from host cells thus acquiring host cell membrane in their new envelope. Any common antigens in this envelope must be glycolipid from the cell of origin. Host membrane proteins are preferentially not taken into new viral envelope, host antigens present being glycolipids which will be common to measles and influenza, when both are derived from vero cells. It is concluded that some oligoclonal bands seen in MS CSF could result from an autoimmune response to host cell glycolipid presented in the envelope of budding viruses which replicate well in oligodendrocytes, the source of myelin. Antiglycolipid immune reactions have been shown to cause demyelination. If enveloped viruses are involved in the aetiology of $M S$, this may be an important mechanism.

PREFERENTIAL GENERATION AND SIGNIFICANCE OF REPEATER F-WAVES (FW) IN MAN

H Modarres-Sadeghi, RJ Guiloff. Westminster Hospital, Charing Cross and Westminster Medical School, London

One thousand five hundred and fifty six conventionally recorded surface $\mathbf{F}$-waves $(\mathrm{Fw}$ ) obtained with 60 supramaximal $1 \mathrm{~Hz}$ stimuli and recorded over the abductor digiti minim were recorded in 30 healthy subjects (mean age $53 \cdot 1$, SD $15 \cdot 1$ ). There was a group mean (SD) of $19.5 \%(8 \cdot 1)$ repeater (rep) waves which was greater than expected by chance. Rep waves occurred randomly during the stimulation period.

Of $1398 \mathrm{Fw}$ shapes, 136 occurred repeatedly, $79 \%$ twice, $18 \%$ thrice and $3 \%$ four times or more; their amplitude and area were higher than that of non-rep $(p<0.001)$ but the latency, conduction velocities (CV) and duration were similar in both groups. This suggests that a greater number of motor units contributes to each rep $\mathrm{Fw}$ and that their associated repeated discharges are not related to motor unit size or $\mathrm{CV}$.

Functional and anatomical arrangements may exist in groups of human motorneurons (MNs), as described in the cat, which make MNs prone to discharge together, more frequently than expected by chance, with antidromic stimulation. The frequency distribution of intervals $(n=160)$ between rep of the same shape showed four peaks, with the majority occurring at intervals of less than $10 \mathrm{~s}$. This suggests heterogeneity in those arrangements, within the groups of MNs generating rep waves.

PREFERENTIAL GENERATION OF F-WAVES BY LARGER MOTORNEURONES IN MAN

RJ Guiloff, H Modarres-Sadeghi. Westminster Hospital, Charing Cross and Westminster Medical School, London

Conventionally recorded surface $\mathrm{F}$-waves
$(F w)(n=1692,33$ subjects $)$ over the abductor digiti minimi muscle (ADM) and evoked by 60 supramaximal stimuli at $1 \mathrm{~Hz}$ had a wide range of conduction velocities (CVs). Single motor unit (MU) F responses to threshold stimulation of the ulnar nerve were recorded with bipolar needle electrodes (BNE); the mean latencies of their fastest associated surface single unit $M$ and $F$ potentials, identified in six subjects, were similar to those of the compound muscle action potential and fastest $F w$, indicating that the fastest Fw adequately indexes the fastest motor fibre of the ulnar nerve. The mean surface unit $F$ amplitude of $34 \mathrm{BNE}$ recorded MUs was similar to that of 158 voluntary MUs recorded in a further eight subjects with a spike triggered averaging technique; an estimated mean of 2-3 MUs per Fw was found by two methods.

The frequency distribution of estimated Fw CVs was shifted towards faster values than expected from available studies of the distribution of CVs in single peripheral nerves; it was also higher than predicted from the expected relation between such distribution and the number of MUs per Fw, if an equal chance of activation and recording is assumed for each MU. There was a significant positive correlation between the frequency of $F$ responses and their $\mathrm{CV}$ in $81 \mathrm{BNE}$ recorded single MUs tested with 200 threshold stimuli ( $\mathrm{n}=0.35, \mathrm{p}<0.01$ ).

These findings are consistent with preferential generation of recurrent responses by larger MUs; it may relate to a lesser chance of antidromic discharge in the smaller motorneurons and to a greater chance of collision of orthodromic (reflex) and antidromic impulses in their axons.

CEREBRAL INSULT-LIKE PROTRACTED SEIZURES IN BENIGN CHILDHOOD EPILEPSY WITH OCCIPITAL PAROXYSMS CP Panayiotopoulos, D Igoe. St Thomas Hospital, London

Benign childhood epilepsy with occipita paroxysms (BCEOP) is a benign condition However, ictal symptoms may imitate grave cerebral insults as shown by a recently seen child and a review of eight out of 16 children with BCEOP who have been followed up by one of us (CPP) for 15 years.

A six year old Chinese girl became suddenly unwell with vomiting. She stood still, urinated and became unresponsive with eyes deviated to one side. On arrival in casualty she was unconscious and hypotonic with limb jerks. Convulsions were controlled with diazepam IV but consciousness was not regained until 6.5 hours after onset of symptoms. An EEG six weeks later demonstrated unilateral occipital paroxysms on eyes closed or elimination of central vision.

The other children (seven girls and one boy) had similar protracted ictal events (more than half an hour to four hours) at a peak age of five years (range 3-7). The cerebral insultlike protracted seizures were nocturnal (seven children) or diurnal (one child), the first (four children) and the only episode (2) or preceded by similar ictal events of short duration (four children). Consciousness was usually impaired or lost (seven children) with hemiconvulsions (two children) or generalised tonic-clonic seizures (four children). The EEG showed unilateral or bilateral occipital spikes which attenuate on eyes open with fixation sensitivity. The prognosis is excellen with infrequent seizures (usually 1-3 and no more than 11 seizures) and complete remission (1-2 years after onset and never after the age of 11 years). Recognition of this benign condition may prevent unnecessary investigations and undue concern.

NON-PAINFUL DISTAI SYMMETRICAI PERIPHERAL NEUROPATHIES IN AIDS

GN Fuller, JM Jacobs, RJ Guiloff. Westminster Hospital, Charing Cross and Westminster Medical School, and Institute of Neurology, London

The most frequent peripheral neuropathies seen in AIDS are distal and symmetrical (DSPN). The majority are painful and appear to be a distinct group associated with CMV infection (PPN). The authors describe here 13 patients with DSPN who did not complain of pain and compare them to 25 patients with PPN seen during the same period.

All 13 were male homosexuals and had AIDS diagnosed for 0-31 months, with a median CD4 count of $13 / \mathrm{u}^{3}$. This group, when compared to patients with PPN, had slower onset symptoms in the arms and weakness significantly more frequently. Two had pes cavus. The group had significantly higher rate of mycobacterium avium intracellulare infection, and lower mean Body Mass Index than 30 AIDS controls. The rate of CMV infection did not differ from controls.

Electrophysiological studies did not differ from those with PPN. When compared to AIDS controls, there was a significant reduction in amplitude of both sensory and motor action potentials, with mild changes in conduction velocity. The CSF was acellular in five studied, with elevated protein in four. Sural nerve from three revealed no inflammation and reduced myelinated fibre $(4,796$ $\left.\mathrm{mm}^{2}\right)$ and unmyelinated axon $\left(22,475 / \mathrm{mm}^{2}\right)$ densities. Unlike PPN, no axonal atrophy was found.

Non-painful DSPN in AIDS are heterogeneous group of neuropathies, including some related to nutritional factors, infections and incidental, perhaps inherited, neuropathies.

THE ROLE OF CT IMAGING IN CLINICAI

EVALUATION OF NEUROMUSCULAR DISORDERS MM Brown, C Thakkar, M Swash. The Royal London Hospital, London

The authors evaluated the role of CT imaging of muscle in the clinical investigation of 20 adult patients with neuromuscular disorders; eight with neurogenic disorders, four with inflammatory myopathies, and eight with limb-girdle and other myopathies. Five CT planes were used; pectoral and upper arms, mid-abdominal, pelvic, mid-thigh and midcalf. Standardised window settings were used for imaging. In the CT images 20 muscles were rated either as normal, or as showing atrophic, "moth-eaten" or "washed-out" abnormalities, and quantitative assessment of the attenuation characteristics of muscles was carried out whenever possible using Hounsfield units. Muscular wasting was graded on a four point scale, and strength was assessed by the MRC scale; 26 muscles were tested clinically. CT/clinical comparison was possible in 11 muscle groups. Moth-eaten change was found in $50 \%$ of muscles examined by CT in inflammatory myopathies, in $20 \%$ of muscles in neurogenic disorders, and in $30 \%$ of muscles in myopathies. Washed-out change was characteristic of myopathies, but was not 
found in the other disorders. The attenuation of abnormal muscles was lowest in the myopathies and inflammatory myopathies. $\mathrm{CT}$ revealed changes in individual components of muscle groups and in certain muscles inaccessible to clinical examination, and thus enhanced the results of clinical muscle testing.

\section{SURFACE EMG IN THE RECORDING OF} FASCICULATIONS

RS Howard, NMF Murray. The National Hospital for Neurology and Neurosurgery, London

The usefulness of multichannel surface recording of fasciculations was evaluated by retrospective study of 116 patients with various neurological disorders. Eight channels of a conventional electroencephalograph were used with plate electrode recordings from the upper and lower arms and legs.

Widespread fasciculations (defined as occurring in five or more of the eight muscle groups) were recorded in $\mathbf{4 8}$ of 54 patients with motor neuron disease, spinal muscular atrophy or post-polio syndrome, but noted on routine clinical examination at presentation in only six. Eleven of 23 patients with peripheral neuropathy or myelopathy had fasciculations in five or more leads compared to one clinically, and three of 39 with other neurological diseases had fasciculations electrically but in only one were they clinically observed.

The method is a non-invasive and sensitive adjunct to clinical examination for detecting fasciculations. Its diagnostic value is limited by the relatively high incidence of fasciculations in neuropathies and myelopathies. However, this study suggests that "false negatives" are rare and that the diagnosis of motor neuron disease should be reconsidered when less than five leads show fasciculations.

THE HYPOCAPNIC APNOEIC THRESHOLD AND ITS IMPORTANCE IN THE CONTROL OF BREATHING

RS Howard, CP Seers, TA Sears. Institute of Neurology, London

The hypocapnic apnoeic threshold may be defined as that $\mathrm{P}_{\mathrm{A}} \mathrm{CO}_{2}$ below which the respiratory motor output is sufficiently inhibited such that the normal respiratory rhythm ceases. Any elevation of this " $\mathrm{CO}_{2}$ threshold" may precipitate an instability in the respiratory rhythm which could lead to prolonged apnoea.

The transition between apnoea and rhythm was studied in anaesthetised rabbits by alter- ing the $\mathrm{CO}_{2}$ threshold with pharmacological agents. Incremental doses of morphine produced an elevation of the $\mathrm{CO}_{2}$ threshold until apnoea supervened. Naloxone, however, led to a dose dependent reduction of the $\mathrm{CO}_{2}$ threshold. Small doses of 5hydroxytrytamine led to a small reduction in the $\mathrm{CO}_{2}$ threshold and with larger doses there was a dose dependent elevation. Progesterone led to a reduction in the $\mathrm{CO}_{2}$ threshold for rhythm generation.

There is considerable evidence of brainstem morphological and pharmacological abnormalities associated with disturbances of central respiratory control in infants and adults. Such abnormalities may contribute to irregularities of the respiratory rhythm by inducing an instability of the central $\mathrm{CO}_{2}$ threshold for transition from apnoea to rhythm generation.

THE EFFECTS OF MORPHINE ON THE

GENERATION OF THE RESPIRATORY RHYTHM

RS Howard, CP Seers, TA Sears. Institute of Neurology, London

Theories describing generation of the respiratory rhythm are typically based either, on the interaction of alternating bursts of inspiratory or expiratory intercostal activity or, upon the termination of a centrally generated inspiratory ramp.

The efferent activity of intercostal inspiratory and expiratory nerve filaments in anaethetised rabbits was recorded. Incremental doses of morphine led to an elevation of the $\mathrm{CO}_{2}$ threshold for rhythm generation and eventually apnoea supervened. Phasic inspiratory and expiratory activity was replaced by tonic firing of the expiratory motor neurons. This tonic expiratory activity responded to increased levels of $\mathrm{CO}_{2}$ by increased discharge frequency of single units or recruitment of new units.

Previous models of rhythm generation cannot account for the access of central chemoreceptor $\mathrm{CO}_{2}$ drive to the respiratory motor neurons in the absence of rhythm. The present results are interpreted on the basis of a model in which $\mathrm{CO}_{2}$ dependent brainstem mediated tonic drives have access to the inspiratory and expiratory pools of motor neurons in the absence of rhythm generation. In this model the rhythmic alternating activity of inspiratory and expiratory motor neurons is sculptured by a phasic interruption of the tonic chemical drives to the respiratory motor neurons. Thus there is a separation between the mechanisms through which the chemical drives gain access to the motor neurons and those concerned in rhythm generation.
THREE CASES OF BILATERAL HORNER'S SYNDROME

SE Smith, SA Smith. St Thomas' Hospital, London

The clinical detection of a sympathetic deficit in the eye is straightforward so long as there is a normal eye for comparison. If the deficit is bilateral, however, its detection is less certain for the classical signs of ptosis, miosis and pupillary re-dilatation lag are hard to discern. Three cases are reported of bilateral sympathetic loss, determined from computerised recordings of re-dilatation time for which a normal range has been constructed. Al measurements were obtained by infrared TV pupillometry.

The three patients were: 1) a 44 year old man with insulin-dependent diabetes mellitus, 2) a 58 year old man with progressive autonomic failure, and 3) a 50 year old woman with anterior spinal artery occlusion yielding sensory and motor signs below C5, 6 . All three showed re-dilatation lag with threequarter recovery times $(4 \cdot 0,4.95$ and $6 \cdot 70 \mathrm{sec}$ respectively) beyond the normal range.

MEASUREMENT OF PULSATILE OCULAR BLOOD FLOW IN PATIENTS WITH NON-ARTERITIC ANTERIOR ISCHAEMIC OPTIC NEUROPATHY CB James, SE Smith. St Thomas' Hospital, London

Non-arteritic anterior ischaemic optic neuropathy (na-AION) results from ischaemia of the retrolamina part of the optic nerve head. Experimental work has shown that the clinical picture of the disease can be produced by occlusion of the posterior ciliary arteries. No evidence of occlusion of these arteries exists in man.

An applanation pneumotonometer linked to a Langham pulsatile ocular blood flow system has been used to measure the pulsatile ocular blood flow (POBF) in 16 patients with unilateral na-AION and 28 normal subjects. Measurements were performed with the subject standing and lying

In the patients with na-AION there was no difference in mean (SD) the POBF between the affected [ $447(42) \mu \mathrm{l} / \mathrm{min}]$ and unaffected eyes [434 (42) $\mu \mathrm{l} / \mathrm{min}$ ]. The POBF was also similar to that found in the normal subjects [433 (32) $\mu \mathrm{l} / \mathrm{min}$ ]. A significant postural decrease in POBF occurred in both groups on assuming the lying position [normal 84 (22) $\mu \mathrm{l} / \mathrm{min}$ : na-AION 82 (24) $\mu \mathrm{l} / \mathrm{min}$ ]

It is characteristic of na-AION that patients often first notice symptoms of visua loss on waking from sleep. The findings suggest that the reduction in POBF associated with lying down may be a contributory factor in the pathogenesis of the disease. If so, this observation may have an important bearing on the treatment of the condition. 